León, J., Montesinos, V., \& Dasí, R. (2021). La Rendición de Cuentas y Responsabilidad Social en los Gobiernos Centrales: Alianza del Pacífico. Contaduría Universidad de Antioquia, 79, 79-102. Doi: https://doi.org/10.17533/udea.rc.n79a04

\title{
La rendición de cuentas y responsabilidad social en los gobiernos centrales: Alianza del Pacífico*
}

Jeimi Maribel León Silva

Universidad de Medellín

jleon@udem.edu.co

Orcid: 0000-0002-2365-1346

Vicente Montesinos Julve

Universitat de Valencia

Vicente.Montesinos@uv.es

Orcid: 0000-0002-5741-017X

Rosa Dasí González

Universitat de Valencia rosa.m.dasi@uv.es

Orcid: 000-003-1787-8138

Este artículo corresponde a uno de los resultados de la tesis de investigación -La divulgación de la información de Responsabilidad Social en las entidades públicas- realizada en la Universidad de Valencia como beneficiaria del programa Pasaporte a la Ciencia, y aporta al foco de trabajo "Sociedad", en el marco del Programa Colombia Científica. 
La rendición de cuentas y responsabilidad social en los gobiernos centrales: Alianza del Pacífico

Resumen: Este artículo tiene como objetivo describir la información que divulgan los gobiernos centrales de los paises de la Alianza del Pacifico (AP), y los roles que estos asumieron en la promoción de la Responsabilidad Social (RS) mediante los informes de rendición de cuentas que fueron presentados por los presidentes de gobierno en el año 2017. El estudio se realizó mediante la técnica de análisis de contenido, utilizando como criterio de clasificación las categorías y los aspectos definidos en las guías para la presentación de informes de sostenibilidad del Global Reporting Initiative (GRI) relacionados con el sector público. Se concluye que los gobiernos centrales divulgan información relacionada con las tres categorías de la RS (económica, social y medioambiental) y han asumido roles de regulador, promotor, colaborador y facilitador para incentivar este tipo de acciones, lo que evidencia que las presiones de los organismos internacionales han influido en las políticas públicas de los países, principalmente en México y Chile.

Palabras clave: Alianza del Pacifico, información gubernamental, Responsabilidad Social, rendición de cuentas, gobiernos centrales, información no financiera.

Accountability and social responsibility in central governments: The Pacific Alliance

Abstract: The purpose of this article is to describe the information disclosed by the central governments of Pacific Alliance (PA) countries, as well as the roles they played in promoting Social Responsibility (SR) through the accountability reports presented by the countries' presidents in 2017. The study was carried out through the content analysis technique, using as classification criteria the categories and aspects defined in the Global Reporting Initiative (GRI) sustainability reporting guidelines for the public sector. It is concluded that central governments disclose information related to the three categories of SR (economic, social and environmental) and have assumed the roles of regulator, promoter, collaborator and facilitator to encourage this type of actions, demonstrating that pressures from international bodies have influenced public policies in the countries, mainly in Mexico and Chile.

Keywords: Pacific Alliance, governmental information, Social Responsibility, accountability, central governments, non-financial information.

\section{A prestação de contas e responsabilidade social nos governos centrais: Aliança do Pacífico}

Resumo: Este artigo tem como intuito descrever a informação que divulgam os governos centrais dos países da Aliança do Pacifico (AP), e os papéis que estes assumiram na promoção da Responsabilidade Social (RS) mediante os relatórios de prestação de contas que foram apresentados pelos presidentes de governo no ano 2017. O estudo foi realizado mediante a técnica de análise de conteúdo, utilizando como critério de classificação as categorias e os aspectos definidos nas guias para a apresentação de relatórios de sustentabilidade do Global Reporting Initiative (GRI) relacionados com o setor público. Conclui-se que os governos centrais divulgam informação relacionada com as três categorias da RS (econômicas, social e do meio ambiente) e tem assumido papéis de regulador, promotor, colaborador e facilitador para incentivar este tipo de ações, o que evidencia que as pressões dos organismos internacionais tem influenciado nas políticas públicas dos países, principalmente no México e no Chile.

Palavras chave: Aliança do Pacifico, informação governamental, Responsabilidade Social, Prestação de contas, governos centrais, informação não financeira.

\section{L’Obligation redditionnelle et la responsabilité sociale dans les gouvernements centraux : l'Alliance du Pacifique}

Résumé: Cet article vise à décrire les informations divulguées par les gouvernements centraux des pays de l'Alliance du Pacifique (AP), et les rôles qu'ils ont assumés dans la promotion de la Responsabilité Sociale (RS) à travers les rapports de responsabilité qui ont été présentés par les présidents de gouvernement en 2017. L'étude a été menée à travers la technique de l'analyse de contenu, en tenant comme critères de classification les catégories et les aspects définis dans les lignes directrices de la Global Reporting Initiative (GRI) sur les rapports de durabilité relatifs au secteur public. Il est conclu que les gouvernements centraux divulguent des informations liées aux trois catégories de RS (économique, sociale et environnementale) et ont assumé des rôles de régulateur, de promoteur, de collaborateur et de facilitateur pour encourager ce type d'actions; ; ce qui montre que les pressions des organisations internationales ont influencé les politiques publiques des pays, en particulier au Mexique et au Chili.

Mots clés: Alliance du Pacifique, information gouvernementale, responsabilité sociale, obligation redditionnelle, gouvernements centraux, information non financière. 
Cont. udea (julio-septiembre, pp. 79-102. (C) Universidad de Antioquia-2021.

\title{
La rendición de cuentas y responsabilidad social en los gobiernos centrales: Alianza del Pacífico
}

\author{
Jeimi Maribel León Silva, Vicente Montesinos Julve y Rosa Dasí González \\ https://doi.org/10.17533/udea.rc.n79a04
}

Primera versión recibida en abril de 2021 - Versión aceptada en junio de 2021

\section{Introducción}

Desde la década de los ochenta los países y los organismos multilaterales vienen realizando esfuerzos para lograr un Desarrollo Sostenible (DS) que permita a las generaciones actuales y futuras un crecimiento económico en equilibrio con el medioambiente y la sociedad (United Nations, 1987, 1992, 2000, 2002, 2012, 2015). Para este objetivo se han establecido unas líneas de actuación encaminadas a dar respuesta a las necesidades de los colectivos sociales, mediante la participación de diferentes actores como gobierno, sector privado y sociedad civil, lo que ha fomentado prácticas socialmente responsables en la economía, el medioambiente y la sociedad, vinculadas al concepto de Triple Bottom Line (TBL) propuesto por Elkington (1998).

Las prácticas de RS fueron adoptadas inicialmente por las empresas para contribuir al DS. Posteriormente, y de manera paulatina, los gobiernos las han ido incorporando, ya sea en su rol externo a través de la promoción de prácticas, e interno, mediante la gestión de las entidades gubernamentales (Hawrysz y Foltys, 2016). Esto ha permitido que la RS alcance un papel más activo, se considere un tema de importancia en las agendas políticas de los países (Albareda et al., 2006, 2007; Lozano et al., 2009; Midttun et al., 2015; Steurer, 2010, 2011) y se hayan logrado avances significativos en las dimensiones económica, social y medioambiental en los niveles administrativos - nacional, regional o local- (Ball et al., 2014) para satisfacer las demandas de la población y de los organismos multilaterales, una búsqueda que trasciende el enfoque empresarial.

Este documento tiene como objetivo identificar la información que divulgan y los roles que asumen los gobiernos centrales de los países de la AP en la promoción de la RS. Para cumplir este propósito, se realiza un estudio de 
caso de los países que conforman la AP, utilizando la técnica de análisis de contenido de los informes anuales de rendición de cuentas que presentaron los presidentes de gobierno en el año 2017, en cumplimiento del mandato constitucional ${ }^{1}$ de cada nación, mediante la categorización de los apartados relacionados con los aspectos y las dimensiones de sostenibilidad contemplados en las directrices de la guía G4 (Global Reporting Initiative, 2013) y en la definición de los roles de gobiernos en la Responsabilidad Social Empresarial (RSE) propuesta por Fox et al. (2002).

Este tipo de estudios en gobiernos centrales permite abordar el tema de la RS en el contexto nacional, identificar las acciones y el papel de los gobiernos, y la gestión realizada por ellos en cuanto a la atención de las necesidades internas y externas, así como de los diversos grupos de interés desde la perspectiva macroeconómica, un tema que ha sido poco desarrollado en el análisis de la sostenibilidad (Gallego-Álvarez et al., 2015) que permite contar con un referente sobre el compromiso de los países latinoamericanos para alcanzar el DS y promover estudios en los gobiernos centrales que son escasos en este campo (Figueira et al., 2018)

El documento se divide en cuatro apartados: en el primero se presenta la revisión de literatura de los gobiernos centrales y la RS; en el segundo se define la metodología del trabajo; en el tercero se relacionan los resultados del estudio, tales como la descripción de la información de sostenibilidad que divulgan las entidades y los roles que asumen los gobiernos con relación a la RS; por último, se presentan las conclusiones y los comentarios generales.

\section{Revisión de literatura}

\section{II.1 Relación entre el gobierno y RS}

La preocupación de muchos países por atender las dificultades de la población ha promovido una serie de iniciativas alrededor del DS. Así, en 1987, con la publicación del informe de la Comisión Mundial de Medio Ambiente y Desarrollo de las Naciones Unidas (United Nations, 1987) "Nuestro Futuro Común" o "Informe Brundtland", se definió el DS como la búsqueda del equilibrio entre las dimensiones económica, social y medioambiental, y esto ha obligado al sector público, al privado y a la sociedad civil a actuar de forma coordinada para lograrlo.

Otra iniciativa importante fue la Cumbre del Milenio de la ONU en el año 2000, donde se concertaron los Objetivos de Desarrollo del Milenio (ODM) encaminados a resolver asuntos económicos, sociales y medioambientales que afectaban en ese momento al planeta, y se insistió en la responsabilidad colec-

1 Constitución Política de la República de Chile (1980) artículo 24; Constitución Política de Colombia (1991), artículo 189; numeral de la Constitución Política del Perú (1993), artículo 118; Constitución Política de los Estados Unidos Mexicanos (1917), artículo 69. 
tiva de todos los agentes interesados para alcanzar el progreso en términos de desarrollo (United Nations, 2000). En el año 2015, ante la imposibilidad de cumplir con las metas de los ODM, se celebró la Cumbre de Desarrollo Sostenible, en la que se adoptó la Agenda 2030 que formula 17 objetivos para avanzar hacia el DS, con el compromiso de los 193 estados miembros (United Nations, 2015).

En simultáneo, se replanteó el papel del Estado, dada su dificultad para atender la totalidad de las demandas de los ciudadanos. Se estableció la perspectiva del Estado Relacional, caracterizado por la motivación de diferentes agentes sociales para participar en la solución de los problemas de la sociedad, desde el enfoque de la corresponsabilidad (Vernis y Mendoza, 2009), y que se vincula con el DS debido a que el éxito de este objetivo no depende exclusivamente de las actuaciones de los gobiernos, sino que precisa de la participación de las otras partes interesadas (sector empresarial y la sociedad civil) para cumplir los compromisos adquiridos, como es el caso de los ODS (Bitar, 2016).

Por lo anterior, el DS se ha convertido en una prioridad para los gobiernos centrales que han impulsado desde sus agendas políticas algunas iniciativas relacionadas con la RSE (Kowszyk et al., 2015), lo que ha permitido que sus actuaciones trasciendan de un papel netamente regulatorio a uno de liderazgo para promoverlas en los diferentes sectores (Fox et al., 2002; Steurer, 2010, 2011), desde una perspectiva interna y externa (Hawrysz y Foltys, 2016).

Sin embargo, el desarrollo de principios y de prácticas ha sido limitado en comparación con los avances en las organizaciones del sector privado (Figueira et al., 2018) y estos aspectos resultan necesarios de incorporar debido a las tendencias actuales de sostenibilidad y a los compromisos para el cumplimiento de los objetivos planteados en la Agenda 2030 (United Nations, 2015) donde los procesos de rendición de cuentas de los gobiernos nacionales resultan esenciales para la implementación y cumplimiento de los Objetivos de Desarrollo Sostenible y la creación de valor social y económico para los grupos de interés (Abhayawansa et al., 2021).

Para Fox et al. (2002), el desafío de los organismos del sector público es identificar los incentivos y prioridades del contexto local y nacional para implementar acciones y convertirse en un actor fundamental de la RS. Además, tienen la obligación y la necesidad de rendir cuentas ante los ciudadanos y otros agentes interesados, como los miembros del parlamento, las entidades de control y los organismos multilaterales, para cumplir con los objetivos planteados en los planes de gobierno de los mandatarios, y otras cuestiones relacionadas con los compromisos y acuerdos suscritos en diferentes escenarios en el contexto nacional, regional y mundial, en nombre de los estados en materia económica, medioambiental, de derechos humanos, trabajo y género. 


\section{II.2 La rendición de cuentas en los gobiernos centrales}

En términos generales, el sector público debe garantizar la administración sostenible de los recursos públicos (Lynch, 2010) y el bienestar de la comunidad a partir de sus necesidades y demandas; además, debe contribuir al fortalecimiento de la confianza de los ciudadanos a través de la evaluación que realizan sobre las funciones del gobierno y las intenciones de los funcionarios públicos para promover el interés público (Mizrahi et al., 2020). Estos elementos han incrementado las presiones a los gobernantes para divulgar información que demuestre avances en los temas relevantes para la sociedad; entre ellos, se destacan los informes relacionados con los compromisos por el medio ambiente, la economía y la sociedad civil, considerando que la presentación de informes no financieros pretenden incrementar la transparencia y reconstruir la confianza en la sociedad (La Torre et al., 2020).

Desde un enfoque teórico, la teoría institucional establece que las organizaciones surgen en contextos altamente institucionalizados, lo que les obliga a incorporar las prácticas y procedimientos definidos que prevalecen en el contexto organizacional y en la sociedad; esto ocasiona que muchos de los procesos y las obligaciones sociales se asuman desde el pensamiento social y en la acción de las organizaciones (Meyer y Rowan, 1977). Esta institucionalización de prácticas es considerada uno de los factores importantes para abordar el papel del gobierno en la RS (Steurer, 2010; Vallentin, 2015), debido a la capacidad que estos tienen para intervenir en estos asuntos y causar efecto en las prácticas de RS en los países (Matten y Moon, 2008). A ello se suma las presiones asociadas a las que están sometidos para cumplir compromisos con la ciudadanía y los organismos multilaterales (United Nations, 2000, 2015), y les obliga a utilizar prácticas de divulgación para evidenciar el cumplimiento de los objetivos.

Para legitimar las acciones y responder a las presiones de diferentes Stakeholders, los gobiernos de cualquier nivel divulgan información sobre su gestión bajo el concepto de la rendición de cuentas "Accountability", la cual está reconocida en la presentación de informes del sector público (Bebbington et al., 2009). La rendición de cuentas ha trascendido los aspectos netamente financieros, para incorporar información de carácter social y medioambiental, como lo señalan Gray et al. (1996), quienes la definen como el deber de las organizaciones de proveer información (no necesariamente un informe financiero) o el cálculo de las acciones por las cuales son responsables.

Esto significa que las entidades presentan información relativa a todas las actividades en las que tengan compromisos, sin limitarse a un tipo en particular. Por lo tanto, los gobiernos también presentan informes de rendición de cuentas de la gestión realizada durante un periodo, de acuerdo con lo establecido en el mandato constitucional de cada país, lo que incluye información 
económica, social y medioambiental, como respuesta a los avances alcanzados en el programa de gobierno o en el plan de desarrollo pactado al inicio de periodo presidencial.

Los Estados también se someten a distintas mediciones para comparar la situación del país con respecto a otras naciones y así demostrar el grado de avance en ciertos indicadores y temas de interés en el contexto internacional, entre los que se destaca el Índice de Desarrollo Humano del Programa de Naciones Unidas para el Desarrollo (PNUD), el Índice de los ODS del Bertelsmann Stiftung, el Índice ODS 2019 para América Latina y el Caribe (2020) y la Red de soluciones de DS; además del Índice de Sostenibilidad Social, calculado cada año por la Fundación de Sostenibilidad Social (SSF por sus siglas en inglés).

Estas mediciones son elaboradas a partir de la información consolidada de los países y de diferentes bases de datos oficiales que evalúan, entre otros, aspectos relacionados con la sostenibilidad. Por lo tanto, la generación y divulgación de información relacionada con las dimensiones de la RS son acciones prioritarias en el contexto actual. Es importante destacar que países como Alemania, Canadá y Suiza están avanzando en la marcos relacionados con la presentación de informes de sostenibilidad, por lo que la divulgación de información de asuntos asociados con aspectos del Triple Botton Line depende en gran medida de la capacidad y voluntad de los gobiernos nacionales (Jones, $2010)$ en sus prácticas de rendición de cuentas.

\section{III.3 La RS en los países en vías de desarrollo}

A partir de la revisión de la literatura sobre RS en el sector público, se observa que, en comparación con los países desarrollados, en los países en vías de desarrollo no abundan investigaciones ni publicaciones académicas sobre el tema en las entidades de gobierno (Ali et al., 2017; Hahn y Kühnen, 2013; León-Silva et al., 2020). Este hecho obedece, por un lado, a que el enfoque inicial de la RS fue relacionado principalmente a las actuaciones de las empresas (Carroll, 1999) y, por otro, al carácter de voluntariedad que había prevalecido, como en el caso de la Comisión Europea (Comisión de las Comunidades Europeas, 2001), debido a que inicialmente la RS surgió a partir de la preocupación de los hombres de negocios para establecer acciones que fueran deseables con los valores de la sociedad (Bowen, 1953), sin que existiera un carácter de obligatoriedad en estos asuntos.

La investigación de la RS en los gobiernos centrales se ha concentrado principalmente en los países de la Unión Europea (UE). Es el caso de los estudios de Albareda et al. (2006, 2007, 2008); Lozano et al. (2009); Steurer (2010, 2011) y Vallentin, (2015). Los análisis se realizan con las agendas políticas de los países del norte, como Europa y Norteamérica, y no se identifica un mayor avance en Latinoamérica ni en África (Idemudia, 2011, 2014). 
Sobre la RS en Latinoamérica se encuentra el trabajo de Kowszyk et al. (2015), que analiza las iniciativas de los gobiernos relacionadas con la RS en las empresas, en los países de la UE y de América Latina y el Caribe (ALC), e identifica los principales avances en la elaboración de planes nacionales de RSE y la integración en las políticas públicas. Este estudio revela el liderazgo de los gobiernos centrales en la promoción de iniciativas que muestran intenciones de vincularse con la economía mundial mediante acuerdos y convenios comerciales, alianzas y tratados internacionales, lo que los ha llevado a adoptar guías y estándares internacionales para alcanzar el DS.

Por esta razón, la presente investigación se concentra en el estudio de caso de los países latinoamericanos que pertenecen a la AP, dada su importancia económica y geopolítica en la región y los avances significativos en materia de RSE (Kowszyk et al., 2015). Además, son países que cuentan con una estructura institucional democrática sólida, con mandatarios elegidos periódicamente, mercados dinámicos y globalizados, y condiciones favorables para la inversión. Esta alianza (AP), es la octava potencia económica y exportadora a nivel mundial; representa el $37 \%$ del Producto Interno Bruto (PIB), el 52\% del comercio total, que atrae el 45\% de la Inversión Extranjera Directa (IED) del ALC (Alianza del Pacífico, s.f.) $)^{2}$.

México, Chile y Colombia ${ }^{3}$ son miembros OCDE y Perú tiene proyectado ingresar en el año 2021. Esta circunstancia resulta interesante, dado que países como España, Italia, Nueva Zelanda, Francia y Países Bajos, entre otros, que están vinculados con este organismo, han implementado iniciativas en materia de RS desde el sector público, a través de iniciativas voluntarias y con normativa vinculada a estos temas.

\section{Metodología}

Con el propósito de identificar la información que divulgan y los roles que asumen los gobiernos centrales, se realizó un estudio de caso en la AP, que está conformada por Chile, Colombia, México y Perú, para la promoción de la RS, utilizando la técnica de análisis de contenido a los informes anuales de los presidentes de los gobiernos centrales de los países de la AP, que fueron presentados en el año $2017^{4}$ como parte del proceso de rendición de cuentas de cada

2 De acuerdo con los datos de organismos internacionales como el Fondo Monetario Internacional (FMI), el Banco Mundial (BM), la Organización Mundial del Comercio (OMC) y la Conferencia de las Naciones Unidas sobre Comercio y Desarrollo (UNCTAD).

3 La OCDE aprobó la adhesión de Colombia en mayo de 2018.

4 Se tomó el año 2017 porque era la información disponible en los cuatro países analizados en las fechas de realización del estudio y en ese año hubo elecciones presidenciales en Chile y en el 2018 en Colombia y México, lo que impedía analizar la gestión de los gobernantes que iniciaron acciones relacionados con la Agenda 2030 y el Acuerdo de París en el año 2015. 
país (Gallego-Álvarez y Quina-Custodio, 2016; Guthrie y Farneti, 2008; Mazzara et al., 2010). Se utiliza la técnica de análisis de contenido cualitativo, definido como un método para la descripción sistemática del material, de acuerdo con la clasificación de las categorías en un marco de codificación (Schreier, 2012), que permite identificar las prácticas de divulgación asociadas a la sostenibilidad de los gobiernos de la AP y al papel que asumen con respecto a este tema para las organizaciones del sector empresarial.

El análisis de contenido es la técnica más empleada en reportes sociales y medioambientales para la recolección de datos asociados con la investigación (Guthrie y Abeysekera, 2006), debido a que el principal objetivo de estos estudios es evaluar lo que divulgan las organizaciones. Además, esta técnica facilita la recopilación de datos, en comparación con otros instrumentos, como las encuestas o las entrevistas (Fifka, 2013). En el caso del sector público, esta técnica se ha utilizado en diversas investigaciones relacionadas con la divulgación de aspectos sociales y medioambientales en los gobiernos locales (Williams, 2015), para lo cual se recurre a los criterios definidos de la guía GRI en sus diferentes versiones (Joseph y Taplin, 2012a; Kaur y Lodhia, 2014; Othman et al., 2017; Williams et al., 2011).

El objeto de análisis son las secciones de páginas del informe anual (Guthrie y Abeysekera, 2006; Unerman, 2000) relacionadas con las acciones de los gobiernos centrales, clasificadas en las categorías económica, social y medioambiental, definidas en la directrices de la guía GRI en la versión G4 (Global Reporting Initiative, 2013), y otros aspectos incorporados en el suplemento para las agencias públicas del GRI (Global Reporting Initiative, 2005). Con esta información se estableció el eje temático para el análisis (Anexo 1). Posteriormente, en los casos que era posible, se clasificaron las iniciativas de acuerdo con los roles de los gobiernos para promover la RSE propuestos por Fox et al. (2002), señalados en el Anexo 2.

\section{Resultados}

En este apartado se presentan los resultados de análisis de contenido de los informes de rendición de cuentas de los presidentes de los países de la AP en el 2017. En la primera parte se exponen las iniciativas relacionadas con las dimensiones económica, social y medioambiental, asociadas con la sostenibilidad que divulgan los gobiernos centrales. En la segunda parte, se presenta el resultado de una clasificación de las iniciativas asociadas con la RS, de acuerdo con los roles definidos por los autores Fox et al. (2002).

En el análisis detallado de la estructura de los informes, Chile y México revelan un mayor nivel de detalle de las acciones realizadas para cumplir con las propuestas consignadas en sus programas de gobierno. También se observan diferencias en la forma de presentar los informes en cuanto a estructura, exten- 
sión y contenido, lo que plantea diferencias importantes en la divulgación de las iniciativas de RS en los cuatro países analizados.

\section{IV.1 Divulgación de información de sostenibilidad en el sector público}

Se identificaron los aspectos de las categorías de la RS que divulgan los gobiernos relacionados con el sector público, como parte de su proceso de gestión para alcanzar el DS, a partir de la relación entre la información que se divulga en los informes de rendición de cuentas de los gobiernos y las guías para la elaboración de los informes de sostenibilidad del GRI como eje temático. A continuación, se presentan algunas iniciativas que divulgan los gobiernos y que contribuyen a conformar la estructura temática del informe.

\section{IV.1.1 Dimensión económica}

En los informes se destaca la preocupación respecto a la situación económica de los países, cuyo contenido ocupa un espacio importante. Entre los aspectos a destacar se encuentran:

- Desempeño económico: se concentra en divulgar el contexto económico de los países y de la región en el último año, y destaca variables macroeconómicas como el crecimiento económico del país, los sectores de la economía de impacto positivo o negativo, el PIB nacional, tasa de desempleo, operaciones de comercio internacional y resultado financiero del Estado, a partir de la explicación de los principales ingresos y gastos del presupuesto. También se destacan las medidas para mejorar el desempeño económico del país, asociadas al manejo del déficit fiscal y a cómo lograr un mayor crecimiento en los siguientes años.

- Presencia en el mercado: en este aspecto los gobiernos (en especial Colombia, Chile y México) mencionan la participación en organismos o alianzas para incentivar las relaciones comerciales y económicas entre diferentes países. También se hace mención del puesto que ocupan en los diferentes rankings que evalúan el desempeño económico. Entre los convenios se destaca la AP, el Acuerdo Transpacífico, Mercosur y la UE, entre otros.

- Prácticas de adquisición: existen iniciativas relacionadas con el pliego de condiciones para las compras públicas, principalmente en Chile y México, que incentivan y otorgan más puntuación en las contrataciones a los proveedores que tengan prácticas socialmente responsables y amigables con el medioambiente y lo establecen como un criterio de selección. También se destacan los incentivos para que pequeñas empresas, campesinos, mujeres y otros grupos minoritarios tengan posibilidad de participar en los procesos de contratación pública. 


\section{IV.1.2 Dimensión medioambiental}

Desde esta dimensión se destaca un compromiso por lograr acciones en torno al cuidado del medioambiente, debido a los problemas que se han generado en estos países por sus economías, basadas en prácticas extractivas, como petróleo y otros minerales (Comisión Económica para América Latina y el Caribe, CEPAL, 2016), lo que ha incrementado los niveles de contaminación y las reclamaciones por parte de la comunidad, y ha contribuido a una mayor participación por parte de las entidades públicas para mitigar el daño ambiental, disminuir la contaminación y garantizar la preservación del medioambiente.

Entre las iniciativas de los países para contribuir al cuidado del medioambiente desde las entidades del sector público se destacan las acciones relacionadas con la energía, el uso y protección de los recursos hídricos, la conservación de la biodiversidad para la protección de especies y la disminución de la emisión de gases invernadero en Chile y en México.

- Energía: en este aspecto se identifican varias iniciativas para el sector público de los gobiernos de Chile y México. Chile promueve programas para la gestión eficiente y acciones amigables con el ambiente, así como un programa de techos solares en entidades públicas, recambio del alumbrado público y una guía de mejoramiento de la gestión eficiente de la energía para fomentar las prácticas de buen uso.

En México se realizaron varios proyectos relacionados con la eficiencia y sustentabilidad energética en municipios, el programa "Ecocasa" para reducir la emisión de gases efecto invernadero y la asignación de recursos financieros para proyectos asociados con las energías renovables.

- Agua: la contaminación de las fuentes hídricas en las regiones, ha logrado que los gobiernos se ocupen de este tema a través de diversas actividades que buscan proteger los océanos y realizan acciones encaminadas a la protección de los recursos hídricos de los territorios. En el caso de Chile se establecieron áreas marinas protegidas para resguardar el ecosistema marino mediante la creación del "Consejo de ministros para el Desarrollo de la Política Oceánica” y del "Santuario de la Naturaleza".

En Colombia se divulga información relacionada con estaciones hidrológicas de monitoreo que trasmiten información sobre su actividad.

En México se divulgan acciones de la entrada en operación de plantas potabilizadoras y el establecimiento de los lineamientos para el otorgamiento de concesiones o asignaciones de agua subterránea salada y programas para la protección de los ecosistemas subterráneos.

El informe de Perú no contiene información de iniciativas enfocadas al sector público para la protección de los recursos hídricos. 
- Biodiversidad: los países de la AP realizan programas para la conservación de la biodiversidad de las especies mediante mecanismos para la protección de aves y animales. En Chile, a través de una normativa específica, se creó el servicio de biodiversidad y de áreas protegidas, así como el reglamento para la elaboración de planes de recuperación, conservación y gestión.

En Colombia se lanzó el proyecto "Colombia BIO", que tiene por objetivo generar condiciones para conocer, conservar y aprovechar sosteniblemente la biodiversidad del país y su potencial.

En México se presentó la "Estrategia Nacional sobre Biodiversidad de México" y su "Plan de Acción al 2030 para la Protección de Especies", y convenios de colaboración y pronunciamientos internacionales de acciones para la protección de las especies.

En Perú se definió el "Reto Biodiversidad", un instrumento de financiamiento para empresas que promuevan el uso sostenible y la puesta en valor de la biodiversidad.

- Emisiones: la firma del Acuerdo de París por los países de la AP ha incentivado iniciativas para la reducción de emisión de gases invernadero. Las entidades del sector público de Chile y de México han establecido proyectos de reducción de gases desde el control de emisiones por parte de algunas industrias y sectores. También han aprobado políticas relacionadas con tecnologías alternativas para el transporte público, e iniciaron acciones para la participación de estos países en el mercado de emisiones. En el caso de Chile, también se implantaron los impuestos verdes para el sector de transporte y de generación.

- Efluentes y residuos: los esfuerzos de los países se enfocan principalmente en programas para el manejo de los diferentes residuos en coordinación con el sector privado. En Chile se destaca el "Programa de Provisión de Residuos Sólidos y de Saneamiento Sanitario", la regulación de contaminantes asociados a la descarga de residuos líquidos a aguas marinas y la vinculación de los productores frente a la responsabilidad de la gestión de los residuos.

En Colombia se menciona un programa relacionado con la gestión de residuos tecnológicos obsoletos en los colegios.

Los informes de México y Perú no registran información explícita sobre este aspecto.

\section{IV.1.3 Dimensión social}

Es la dimensión que presenta el mayor nivel de detalle en los informes de los presidentes, debido a que corresponde a los principales objetivos de los gobiernos centrales por las dificultades sociales que sufren los habitantes de Latinoamérica (Comisión Económica para América Latina y el Caribe, CEPAL, 2016; 
Organización para la Cooperación y el Desarrollo Económico, OCDE, 2017). Se destaca la información sobre avances en la disminución de la pobreza, garantía de derechos humanos para diferentes grupos de población en condiciones de vulnerabilidad (niños, mujeres, población LGTBI), educación, salud, empleo y la provisión de servicios públicos básicos. Adicionalmente, presentan aspectos relevantes que involucran acciones directamente relacionadas con la labor del Estado para la promoción y participación en dicho proyecto.

- Empleo: se destacan las acciones para incentivar las prácticas laborales que conciernen a los funcionarios públicos. En Chile la normativa está relacionada con el diseño de estructuras del personal y los beneficios entregados a funcionarios de diferentes entidades, así como la ampliación de plazas para funcionarios en distintos sectores, de programas de formación en temas de Estado y la implementación del Instructivo Presidencial №001 de 2015 sobre buenas prácticas laborales para los funcionarios del Estado y las acciones para la "Agenda de Probidad y Transparencia" del gobierno en el portal de empleos públicos.

En los demás países de la AP no se evidencian acciones concretas relacionadas con el empleo de funcionarios públicos.

- Capacitación: de acuerdo con las necesidades del servicio, las entidades gubernamentales de Chile se han preocupado por la formación y capacitación de los funcionarios públicos. Se muestra un grado de apropiación en temas sensibles para la atención de los ciudadanos, como violación de derechos y discriminación de personas en condición de vulnerabilidad.

En el caso de México, el gobierno diseñó un portal de capacitación, adiestramiento y productividad laboral para entidades federativas, que ofrece a los servidores públicos el desarrollo y fortalecimiento de habilidades y competencias conceptuales sobre perspectiva de género, política pública de igualdad, prevención y atención del acoso sexual, no discriminación y derechos humanos.

Colombia y Perú no presentan información sobre acciones encaminadas a la formación del capital humano de las entidades del sector público.

- Diversidad e igualdad de oportunidades: Chile y México divulgan acciones relacionadas con este tema que no solo afectan al sector público y tienen incidencia en la promoción de la igualdad. En Chile, en el marco de los ODS y la instalación de la Mesa del Sector Público de Buenas Prácticas Laborales con Enfoque de Género, se diseñó y formuló el "Plan de Igualdad entre Mujeres y Hombres 2017-2030”, además de cursos y campañas tendientes a incentivar la igualdad de condiciones y oportunidades para la población desde la perspectiva de género, como el programa "Mujer Proveedora" para participar en los procesos de contratación con el Estado.

En México se está ejecutando el "Programa Nacional para la Igualdad de Oportunidades y no Discriminación Contra las Mujeres (PROIGUALDAD)”, y se 
realizó la primera sesión abierta del "Sistema Nacional para la Igualdad Entre Mujeres y Hombres".

En Colombia y Perú no se obtuvo información sobre este aspecto.

No discriminación: este aspecto resulta relevante y se evidencian acciones en los países en diversos temas: género, población inmigrante, comunidad LGTBI.

Chile es el país abanderado en este tema en materia normativa y de gestión. Entre las acciones adelantadas se destacan: el "Taller de Capacitación en Enfoque de Género para la Administración Pública” bajo el plan de modernización del acuerdo de asociación de Chile y la UE del "Programa Regional para la Cohesión Social con América Latina (EUROSOCIAL)", el "Observatorio de Participación Ciudadana y no Discriminación Arbitraria", la Ley 20609 de rechazo y sanción de todo acto discriminatorio de carácter arbitrario, entre otros.

México también ha adelantado acciones en este tema en materia normativa, así como programas para acabar con la discriminación. Entre ellos se destaca la "Instrucción Presidencial IP-321,1" para articular acciones que promuevan el empoderamiento de las mujeres, la lucha contra la discriminación por razones de género, y el fortalecimiento de los sistemas estatales de igualdad, de prevención, atención, sanción y erradicación de la violencia contra las mujeres.

En Perú se identifican acciones para la integración de la persona con discapacidad "CONADIS" y la puesta en marcha de una "Plataforma Virtual Contra el Racismo".

Colombia no cuenta con acciones específicas al respecto.

- Lucha contra la corrupción: este aspecto está contemplado en la Guía GRI G4 y en el suplemento de las entidades públicas, y se considera crucial para Latinoamérica debido a los altos niveles de percepción de corrupción que han llevado al estancamiento del crecimiento de la región y debilitado la confianza de la ciudadanía (Corporación Latinobárometro, 2018). Frente a este tema, cada país ha trabajado en diversas estrategias para eliminar actos corruptos con las entidades del Estado.

En Chile se implementó el "Sistema de Prevención de Lavado de Dinero y Anticorrupción en el Sector Público" y se hizo seguimiento a la implementación de la "Convención Interamericana contra la Corrupción".

En México se realizó una reforma constitucional para crear el "Sistema Nacional Anticorrupción”, una instancia de coordinación para la prevención, detección y sanción de hechos de corrupción, y fortalecimiento de la fiscalización y control de recursos públicos; y se aprobó el "Compendio de las mejores prácticas para el uso de datos abiertos contra la corrupción”.

En Perú se tomaron medidas normativas con la emisión del Decreto Legislativo 1295, que ordena separar del cargo a aquellos trabajadores públicos que hayan cometido delitos de corrupción y el Decreto Legislativo 1307, que modi- 
fica el Código Procesal Penal para hacer más eficaz la persecución y sanción de delitos de corrupción de funcionarios.

En el informe de Colombia no se presenta información sobre este tema, a pesar del creciente número de casos asociados con este delito en los últimos años.

- $\quad$ Norma de calidad del servicio: debido a la necesidad de fortalecer la labor del Estado en el cumplimiento de las necesidades de los ciudadanos y los compromisos a nivel regional y mundial, los países han realizado varias acciones para mejorar la prestación del servicio en las entidades públicas.

Chile ha establecido diferentes iniciativas para fortalecer y modernizar el sector público con orientación hacia el usuario, entre las que se destacan: el "Programa de Formación a los Encargados de la Ejecución y Planificación de las Políticas de Participación en el Estado", la creación del "Comité de Innovación Pública" y del "Laboratorio de Gobierno".

Por su parte, México estableció varias acciones relacionadas con el mejoramiento de la función del sector público. Definió un programa de mejora regulatoria en la Administración Pública Federal (APF), simplificó trámites prioritarios y puso en marcha la estrategia transversal "Gobierno cercano y moderno".

Las acciones realizadas en Perú corresponden a tareas de tipo operativo para agilizar los procesos y las diligencias ante el Estado, como la creación de la herramienta intersectorial "Dime tu traba" e "Invierte.pe".

En el informe presentado por Colombia no se evidencian acciones relacionadas con la calidad del servicio en las entidades públicas.

\section{IV.2 Análisis de los roles de los gobiernos centrales en RS}

Como se ha señalado, el documento del BM Public Sector roles in strengthening corporate social responsibility: a baseline study, publicado por Fox et al. (2002), establece un punto de partida en el estudio de la influencia de los gobiernos en la RSE, al determinar que no solo cumplen el rol de reguladores de las prácticas socialmente responsables de los diferentes agentes, sino que también pueden promoverlas, facilitarlas y colaborar para alcanzar el propósito común del DS.

A partir de esta clasificación (Anexo 2), se tomaron secciones de los informes de rendición de cuentas para establecer los roles asumidos por los gobiernos centrales de la AP en materia de RSE.

El rol de regulador es considerado por los gobiernos el más importante para incentivar las actuaciones de los otros agentes sociales, y mediante la normativa han logrado acciones encaminadas a las prácticas socialmente responsables de todos los sectores (Albareda et al., 2007; Steurer et al., 2012).

En los informes, se observa que la regulación es el rol principal para los estados frente a acciones de la RSE. No solamente los gobiernos han incentivado las buenas actuaciones de las entidades del sector público para alcanzar un 
nivel de desarrollo mediante el cumplimiento de estándares básicos, también han asumido una serie de compromisos en los acuerdos con los organismos multilaterales y convenios con otros países, que los obliga a cumplir aspectos relacionados con la protección del medioambiente, las condiciones laborales, la no discriminación, los derechos de los niños y las mujeres, prácticas laborales y la lucha contra la corrupción, entre otros.

En general, se observa un interés generalizado por contribuir con acciones para alcanzar el crecimiento económico proyectado, incentivar la economía, el cuidado del medioambiente, la lucha contra la discriminación, los derechos laborales y la protección de los pueblos indígenas, entre otros.

El rol de regulador coincide con el papel que ejerce la Unión Europea; especialmente, con la expedición de la Directiva 2104/95 en el año 2014 que estableció requisitos obligatorios para la presentación de informes no financieros en los Estados europeos para mejorar la transparencia e incrementar la confianza en la sociedad y representan cambios en la divulgación de las acciones sociales, medio ambientales y económicas de las empresas de la región (La Torre et al., 2020).

En el rol de facilitador el gobierno interviene mediante herramientas o iniciativas que permiten al sector privado adoptar prácticas de RS, con el fin de mejorar aspectos sociales y medioambientales; sin embargo, cumple un papel secundario, dado que solo manifiesta su interés para que otros agentes sociales realicen esas prácticas desde la voluntad, contando con el apoyo del gobierno para llevar a cabo esas iniciativas, como la creación de sistemas y programas que establezcan criterios para que las entidades del sector público y del sector privado generen prácticas socialmente responsables.

El rol de Estado como colaborador se ha dado especialmente a través de iniciativas que permiten establecer relaciones de asociación con el sector privado, con otras entidades y con la sociedad civil, sobre temas que promueven prácticas de RSE, mediante alianzas público-privadas que en los últimos años han permitido el desarrollo de varios proyectos en el sector privado, con el apoyo de los gobiernos sobre temas que incluyen medioambiente, incentivos a la economía, infraestructura y acciones de protección de los derechos humanos, entre otros.

En los países de la AP ha adquirido fuerza la colaboración público-privada y de organismos multilaterales a través de la ejecución de proyectos relacionados con las dimensiones de la DS, debido a que a los Estados no les ha sido posible cumplir todas las demandas de las ciudadanías. Esta colaboración se refleja principalmente en iniciativas de infraestructura y de mejoramiento de las condiciones de vida de la población.

En el escenario de promotor, el rol del gobierno se manifiesta a través del apoyo político y del respaldo del sector público a las iniciativas de RSE, mediante un conjunto de actividades que impulsan actividades socialmente res- 
ponsables, como reconocimientos por las buenas prácticas, condiciones para la contratación pública u otros incentivos que permiten un mejor acercamiento por parte del sector privado a prácticas vinculadas con la RS. Este rol se refleja en iniciativas que permiten a los proveedores del Estado buenas prácticas en producción y en procesos de contratación, mediante concursos y procesos de certificación de las empresas de prácticas socialmente responsables en materia de producción de artículos con eficiencia energética, no discriminación de la mujer y de la población en condición de discapacidad, entre otros.

\section{Conclusiones}

La RS ha tomado importancia para los gobiernos debido a la urgencia de acciones concretas para alcanzar el DS. Aunque inicialmente solo fue considerada por el sector privado, los gobiernos centrales han empezado a contemplarla mediante el impulso de los acuerdos y los compromisos internacionales adquiridos en materia de crecimiento económico, protección del medioambiente y garantía de los derechos sociales, a partir de un esquema relacional que involucra programas con el sector privado y con la sociedad civil. Aunque cada gobierno tiene diferentes ritmos para la implementación de políticas y prácticas relacionadas con la sostenibilidad, que dependerán de las condiciones políticas, sociales y económicas de los países (Abhayawansa et al., 2021).

En el examen de los informes anuales de los presidentes de los países que conforman la AP, se observan diferencias en cuanto a estructura, tamaño y contenido de los documentos. Se evidencia que Chile y México realizan un esfuerzo por reportar de manera detallada las acciones realizadas durante el periodo del informe, lo que permite al usuario de la información conocer de forma precisa los logros y avances del presidente y su gabinete, de acuerdo con las metas planteadas al inicio del mandato, aunque tanto contenido pueda generar apatía en los ciudadanos y demás grupos de interés. La mayor divulgación de información de prácticas de sostenibilidad también está asociada con el relacionamiento de organizaciones y compromisos internacionales vinculados con la OCDE.

En el caso de Colombia el informe es breve y está centrado en los temas principales del Plan Nacional de Desarrollo (PND). Un caso similar ocurre con el informe de Perú, el cual, aunque utiliza imágenes y detalla algunas acciones sobre temas puntuales, presenta un contenido aún menor. En tal sentido, los informes de Colombia y Perú no permiten identificar la totalidad de las actuaciones de sus gobiernos, ni las gestiones realizadas en materia de RSE, pese a que todos los países de la AP han adoptado compromisos con los ODS y con el cambio climático. En el caso de Perú, se puede destacar la crisis política del gobierno del presidente Pedro Pablo Kuczynski desde su toma de posesión en el 2016, afectando el cumplimiento de su plan de gobierno (República del Perú, 2017) y que concluyó con la destitución del presidente en marzo de 2018. 
En cuanto a la divulgación de la información sobre RS por parte del sector público, se evidencia que las entidades de gobierno realizan acciones que coinciden con algunos aspectos puntuales de las dimensiones (económica, medioambiental y social) contemplados en las guías para los reportes de sostenibilidad definidos por el GRI. En el caso de la dimensión económica, todos los países divulgan información relacionada con la situación del país a través de indicadores macroeconómicos, en los que resaltan acciones para reactivar la economía, y mencionan acuerdos comerciales y prácticas de adquisición transparentes e inclusivas para beneficio de campesinos y mujeres, como las adoptadas por Chile y México.

En la dimensión medioambiental, se destacan las acciones relacionadas con el propósito de reducir las emisiones de gases efecto invernadero por los compromisos con el Acuerdo de París y la ONU; y en la dimensión social, varias acciones relacionadas con prácticas laborales adecuadas para que los empleados públicos presten un buen servicio a los ciudadanos a través de capacitaciones, así como para actualizar prácticas de las entidades del sector público en diversos aspectos relacionados con los derechos humanos y la gestión pública.

Los países de la AP asumen diversos roles (regulador, facilitador, colaborador y promotor) en la implementación de la RS en el sector público y privado, mediante el impulso de iniciativas relacionadas con las tres dimensiones de la sostenibilidad. Entre estos se destaca el rol de regulador, en el que el Estado propone acciones para gestionar en el sector económico, medioambiental y social, principalmente en los temas relativos a los compromisos pactados con organismos multilaterales y otros países, como el cambio climático y el cumplimento de la agenda 2030.

Se evidencia que Chile y México son los países que más divulgan información relacionada con acciones para incentivar la RS, y que su papel no se limita a un aspecto netamente normativo, sino que se extiende al impulso, promoción y participación en diferentes proyectos e iniciativas para lograr el DS, conocida como regulación suave (Steurer, 2010, 2011). En el caso de Colombia y Perú se obtuvo información limitada por el diseño y la poca profundidad del informe, lo que impidió conocer las iniciativas que promueven estos países, si se tiene en cuenta que sus Planes Nacionales de Desarrollo (PND) están encaminados al cumplimiento de la agenda 2030, que impulsa las tres dimensiones del DS (Centro Nacional de Planeamiento Estratégico Perú hacia el 2021, 2016; Departamento Nacional de Planeación, 2014).

Los gobiernos nacionales, al igual que las organizaciones, están sometidos a presiones internas y externas. Por un lado, la ciudadanía y los organismos de control demandan resultados de gestión adecuada y sostenible de los recursos públicos financieros y naturales, así como de atención de las necesidades de la población. Por otro lado, las instituciones internacionales y los efectos de la 
globalización han generado una serie de acciones conjuntas en la mayoría de los países, que han derivado en compromisos para alcanzar metas comunes, entre las que se destaca la garantía de los derechos humanos, el cuidado del medioambiente, crecimiento económico y relaciones comerciales.

Lo anterior ha llevado a que las autoridades gubernamentales promuevan políticas públicas y otras iniciativas de gestión para contribuir al DS, lo cual es más evidente en los países de la OCDE (México y Chile), quienes divulgan información más detallada de las acciones relacionadas con la economía, el medioambiente y la sociedad, que garantiza el cumplimiento de directrices en estos asuntos, a diferencia de Colombia y Perú que, según los informes, no presentan un análisis sobre avances y resultados en la materia. Es importante destacar que la divulgación de información de prácticas de sostenibilidad es similar a los resultados obtenidos en el estudio de León-Silva et al. (2020) relacionada con la divulgación de información de sostenibilidad para los gobiernos locales de la AP.

Se considera que las conclusiones de este estudio son relevantes porque contribuyen a la investigación de la sostenibilidad del sector público en varios aspectos: en primer lugar, el trabajo trata sobre gobiernos centrales de países en vías de desarrollo que no han sido estudiados a profundidad desde el punto de vista académico en el tema de sostenibilidad, dadas las características particulares de estos países; en segundo lugar, se desarrolla el trabajo desde la perspectiva macroeconómica, al analizar las acciones del gobierno central de cada uno de los países de la AP, lo que permite contar con un panorama sobre la ruta nacional para aportar al DS, teniendo en cuenta que la mayoría de los trabajos de sostenibilidad en el sector público se han centrado principalmente en gobiernos locales; por último, este trabajo aborda la sostenibilidad en los gobiernos nacionales, en las dimensiones interna y externa, y evalúa las acciones que buscan promover la RS en el sector privado, así como las iniciativas socialmente responsables que emprenden las entidades públicas como contribución al DS.

Sin embargo, los alcances son limitados debido a la ausencia de información de Colombia y Perú, relacionada con las acciones de sus gobiernos sobre RS en el período presidencial, y dificulta la formulación de conclusiones para el conjunto de países. Adicionalmente, en la revisión de literatura, no se encontró información sobre trabajos previos que analicen los gobiernos centrales desde la perspectiva de la rendición de cuentas, lo que limitó aún más la posibilidad de comparar resultados.

\section{Referencias bibliográficas}

Abhayawansa, S., Adams, C. A. \& Neesham, C. (2021). Accountability and governance in pursuit of Sustainable Development Goals: conceptualising how governments create value. Accounting, Auditing and Accountability Journal, 34(4), 923-945. https://doi. org/10.1108/AAAJ-07-2020-4667 
La Rendición de Cuentas y Responsabilidad Social en los Gobiernos Centrales: Alianza del Pacífico

Albareda, L., Tencati, A., Lozano, J. M. \& Perrini, F. (2006). The government's role in promoting corporate responsibility: a comparative analysis of Italy and UK from the relational state perspective. Corporate Governance: The International Journal of Business in Society, 6(4), 386-400. https://doi.org/10.1108/14720700610689504

Albareda, L., Lozano, J. M. \& Ysa, T. (2007). Public policies on corporate social responsibility: The role of governments in Europe. Journal of Business Ethics, 74(4), 391-407. https:// doi.org/10.1007/s10551-007-9514-1

Albareda, L., Lozano, J. M., Tencati, A., Midttun, A. \& Perrini, F. (2008). The changing role of governments in corporate social responsibility: drivers and responses. Business Ethics: A European Review, 17(4), 347-363. https://doi.org/10.1111/j.14678608.2008.00539.x

Ali, W., Frynas, J. G. \& Mahmood, Z. (2017). Determinants of Corporate Social Responsibility (CSR) Disclosure in Developed and Developing Countries: A Literature Review. Corporate Social Responsibility and Environmental Management, 24, 273-294. https://doi.org/10.1002/csr.1410

Alianza del Pacífico. (2018, 1 de diciembre). What is the Pacific Alliance? https:// alianzapacifico.net/en/what-is-the-pacific-alliance/

Ball, A., Grubnic, S. \& Birchall, J. (2014). Sustainability accounting and accountability in the public sector. In J. Bebbington, J. Unerman \& B. O 'Dwyer (Eds.), Sustainability Accounting and Accountability (2nd editio, pp. 176-195). Routledge.

Bebbington, J., Higgins, C. \& Frame, B. (2009). Initiating sustainable development reporting: evidence from New Zealand. In Accounting, Auditing \& Accountability Journal (Vol. 22, Issue 4). https://doi.org/10.1108/09513570910955452

Bitar, S. (2016). Las tendencias mundiales y el futuro de América Latina. Serie Gestión Pública, 85, 70. http://repositorio.cepal.org/bitstream/handle/11362/40788/1/S1600740_es.pdf

Bowen, H. (1953). Social responsibilities of the businessman. University of Iowa Press.

Carroll, A. B. (1999). Corporate Social Responsibility: Evolution of a Definitional Construct. Business \& Society, 38(3), 268-295. https://doi.org/10.1177/000765039903800303

Centro Nacional de Planeamiento Estratégico Perú hacia el 2021. (2016). Plan Estratégico de Desarrollo Nacional Actualizado. file://C:/Users/Marcia/Downloads/PEDN-2021-1507-2016-RM-138-2016-PCM.pdf

Centro de los Objetivos de Desarrollo Sostenible para América Latina y el Caribe- CODS (2020) Índice ODS 2019 para América Latina y el Caribe. Centro de los Objetivos de Desarrollo Sostenible para América Latina y el Caribe: Bogotá, Colombia.

Comisión de las Comunidades Europeas. (2001). Libro Verde: Fomentar un marco europeo para la responsabilidad social de las empresas (p. 35).

Comisión Económica para América Latina y el Caribe (CEPAL). (2016). Horizontes 2030: La igualdad en el centrol del desarrollo sostenible. Síntesis.

Corporación Latinobárometro. (2018). Informe 2018. In Informe 2018 (Vol. 1). http://www. latinobarometro.org/lat.jsp

Departamento Nacional de Planeación. (2014). Bases del Plan Nacional de Desarrollo 20142018 “Todos por un nuevo país” (Vol. 2). https://goo.gl/wuDSYZ 
Elkington, J. (1998). Partnerships from Cannibals with Forks: The Triple Bottom Line of 21 st-Century Business. Environmental Quality Management, 8(1), 37-51. https://doi. org/10.1002/tqem.3310080106

Fifka, M. S. (2013). Corporate Responsibility Reporting and its Determinants in Comparative Perspective - a Review of the Empirical Literature and a Meta-analysis. Business Strategy and the Environment, 22(1), 1-35. https://doi.org/10.1002/bse.729

Figueira, I., Domingues, A. R., Caeiro, S., Painho, M., Antunes, P., Santos, R., Videira, N., Walker, R. M., Huisingh, D. \& Ramos, T. B. (2018). Sustainability policies and practices in public sector organisations: The case of the Portuguese Central Public Administration. Journal of Cleaner Production, 202, 616-630. https://doi. org/10.1016/j.jclepro.2018.07.244

Fox, T., Ward, H. \& Howard, B. (2002). Public sector roles in strengthening corporate social responsibility: A baseline study. The World Bank, October, 1-33.

Gallego-Álvarez, I., Galindo-Villardón, M. P. \& Rodríguez-Rosa, M. (2015). Analysis of de Sustainable Society Index Worldwide: A Study from the Biplot Perspective. Social Indicators Research, 120(1), 29-65. https://doi.org/10.1007/s11205-014-0579-9

Gallego-Álvarez, I. \& Quina-Custodio, I. A. (2016). Disclosure of corporate social responsibility information and explanatory factors. Online Information Review, 40(2), 218-238. https://doi.org/10.1108/OIR-04-2015-0116

García, I. M., Gallego, I. \& Rodríguez, L. (2013). Información y Responsabilidad Social de las Entidades Públicas Contribución a la sostenibilidad global (1 ra edición). Editorial Académica Española.

Global Reporting Initiative. (2005). Sector Supplement for Public Agencies: Pilot Version 1.0 (Issue March). https://www.far.se/contentassets/fc9a7e4c15cf45c98fd838069253f8d2/ publicagenciessectorsupplementpilot.pdf

Global Reporting Initiative. (2013). G4 Sustainability Reporting Guidelines. In Global Reporting Initiative. https://doi.org/10.1590/S0101-60832006000700003

Gray, R., Owen, D. \& Adams, C. A. (1996). Accounting \& accountability: changes and challenges in corporate social and environmental reportingle. Prentice Hall.

Guthrie, J. \& Abeysekera, I. (2006). Content analysis of social, environmental reporting: what is new? Journal of Human Resource Costing \& Accounting, 10(2), 114-126. https://doi.org/10.1108/14013380610703120

Guthrie, J. \& Farneti, F. (2008). GRI sustainability reporting by Australian public sector organizations. Public Money \& Management, 28(6), 361-366. https://doi.org/10.1111/ j.1467-9302.2008.00670.x

Hahn, R. \& Kühnen, M. (2013). Determinants of sustainability reporting: A review of results, trends, theory, and opportunities in an expanding field of research. Journal of Cleaner Production, 59, 5-21. https://doi.org/10.1016/j.jclepro.2013.07.005

Hawrysz, L. \& Foltys, J. (2016). Environmental Aspects of Social Responsibility of Public Sector Organizations. Sustainability, 8(19), 1-10. https://doi.org/10.3390/su8010019

Idemudia, U. (2011). Corporate social responsibility and developing countries: moving the critical CSR research agenda in Africa forward. Progress in Development Studies, 11(1), 1-18. https://doi.org/10.1177/146499341001100101 
La Rendición de Cuentas y Responsabilidad Social en los Gobiernos Centrales: Alianza del Pacífico

Idemudia, U. (2014). Corporate Social Responsibility and Development in Africa: Issues and Possibilities. Geography Compass, 8(7), 421-435. https://doi. org/10.1177/097168589800400207

Jones, H. (2010). Sustainability reporting matters. In Association of Chartered Certified Accountants (ACCA). http://www.accaglobal.com/content/dam/acca/global/PDFtechnical/sustainability-reporting/tech-tp-srm.pdf

Joseph, C. \& Taplin, R. (2012). International initiatives influence on local government sustainability web-disclosures. Social Responsibility Journal, 8(4), 589-602. https:// doi.org/10.1108/17471111211272561

Kaur, A. \& Lodhia, S. K. (2014). The state of disclosures on stakeholder engagement in sustainability reporting in Australian local councils. Pacific Accounting Review, 26(12), 54-74. https://doi.org/10.1108/PAR-07-2013-0064

Kowszyk, Y., Besnier, E., Haddad, I., Maher, R. \& Meneses, G. (2015). La Responsabilidad Social Empresarial en el contexto de la relación entre la Unión Europea y la Comunidad de Estados Latinoamericanos y Caribeños. https://doi.org/http://dx.doi. org/10.12858/0615es3

La Torre, M., Sabelfeld, S., Blomkvist, M. \& Dumay, J. (2020). Rebuilding trust: sustainability and non-financial reporting and the European Union regulation. Meditari Accountancy Research, 28(5), 701-725. https://doi.org/10.1108/MEDAR-06-2020-0914

León-Silva, J. M., Dasí-González, R. M. \& Montesinos-Julve, V. (2020). Disclosure of sustainability information in the local governments of the pacific alliance. Lex Localis, 18(3), 557-578. https://doi.org/10.4335/18.3.557-578(2020)

Lozano, J., Albareda, L., Ysa, T., Roscher, H. \& Marcuccio, M. (2009). Governments and corporate social responsibility: Public policies beyond regulation and voluntary compliance (1st Edition, p. 195). Palgrave MacMillan. https://doi.org/10.1017/ CB09781107415324.004

Lynch, B. (2010). An examination of environmental reporting by Australian state government departments. Accounting Forum, 34(1), 32-45. https://doi.org/10.1016/j. accfor.2009.11.001

Matten, D. \& Moon, J. (2008). "Implicit" and "Explicit" CSR: A Conceptual Framework for a Comparative Understanding of Corporate Social Responsibility. Academy of Management Review, 33(2), 404-424.

Mazzara, L., Sangiorgi, D. \& Siboni, B. (2010). Public strategic plans in Italian local governments: A sustainability development focus? Public Management Review, 12(4), 493-509. https://doi.org/10.1080/14719037.2010.496264

Meyer, J. W. \& Rowan, B. (1977). Institutionalized Organizations: Formal Structure as Myth and Ceremony. American Journal of Sociology, 83(2), 340-363. https://doi. org/10.1086/226550

Midttun, A., Gjølberg, M., Kourula, A., Sweet, S. \& Vallentin, S. (2015). Public Policies for Corporate Social Responsibility in Four Nordic Countries: Harmony of Goals and Conflict of Means. Business and Society. https://doi.org/10.1177/0007650312450848

Mizrahi, S., Cohen, N. \& Vigoda-Gadot, E. (2020). Government's social responsibility, citizen satisfaction and trust. Policy and Politics, 20(20), 443-460. https://doi.org/10. 1332/030557320X15837138439319 
Organización para la Cooperación y el Desarrollo Económicos (OCDE). (2017). Panorama de las Administraciones Públicas América Latina y el Caribe 2017 (2da ed.). Éditions OCDE. https://doi.org/http://dx.doi.org/9789264266391-es

Othman, R., Nath, N. \& Laswad, F. (2017). Sustainability Reporting by New Zealand's Local Governments. Australian Accounting Review, 27(3), 315-328. https://doi.org/10.1111/ auar.12153

República del Perú. (2017). Memoria de Gobierno República del Perú (Issue 1).

Schreier, M. (2012). Qualitative content analysis in practice (First Edit). SAGE Publications Inc.

Steurer, R. (2010). The role of governments in corporate social responsibility: characterising public policies on CSR in Europe. Policy Sciences, 43(1), 49-72. https://doi.org/10.1007/ s11077-009-9084-4

Steurer, R. (2011). Soft Instruments, Few Networks: How "New Governance" Materializes in Public Policies on Corporate Social Responsibility Across Europe. Environmental Policy and Governance, 21(4), 270-290. https://doi.org/10.1002/eet.575

Steurer, R., Martinuzzi, A. \& Margula, S. (2012). Public Policies on CSR in Europe: Themes, Instruments, and Regional Differences. Corporate Social Responsibility and Environmental Management, 19(4), 206-227. https://doi.org/10.1002/csr.264

Unerman, J. (2000). Methodological issues - Reflections on quantification in corporate social reporting content analysis. Accounting, Auditing \& Accountability Journal, 13(5), 667-681. https://doi.org/10.4324/9780203212813_chapter_1

United Nations. (1987). Report of the World Commision on Environement and Development: Our Common Future. In United Nations. https://doi.org/10.2307/2621529

United Nations. (1992). The Rio declaration on environment and development. United Nations Conference on Environment and Development.

United Nations. (2000). Millenium Declaration (Issue 52/2). https://doi.org/http://www. undp.org/content/undp/en/home/mdgoverview.html

United Nations. (2002). Plan of Implementation of the World Summit on Sustainable Development. In The United Nations Conference on Environment and Development.

United Nations. (2012). The future we want (Issue September).

United Nations. (2015). Transforming our world: the 2030 agenda for sustainable development.

Vallentin, S. (2015). Governmentalities of CSR: Danish Government Policy as a Reflection of Political Difference. Journal of Business Ethics, 127(1), 33-47. https://doi.org/10.1007/ s10551-013-1703-5

Vernis, A. \& Mendoza, X. (2009). Una aproximación a la conceptualización del nuevo rol del Estado: el Estado relacional. Revista del CLAD Reforma y Democracia, 44, 115-144.

Williams, B. R. (2015). The local government accountants' perspective on sustainability. Sustainability Accounting, Management and Policy Journal, 6(2), 267-287. https://doi. org/10.1108/SAMPJ-07-2014-0043

Williams, B., Wilmshurst, T. \& Clift, R. (2011). Sustainability reporting by local government in Australia: Current and future prospects. Accounting Forum, 35(3), 176-186. https:// doi.org/10.1016/j.accfor.2011.06.004 
La Rendición de Cuentas y Responsabilidad Social en los Gobiernos Centrales: Alianza del Pacífico

Anexo 1. Categorías y aspectos de los informes de sostenibilidad del GRI

\begin{tabular}{|c|c|c|}
\hline Categoría & Guía G4 & Suplemento guía GRI entidades públicas \\
\hline \multirow{4}{*}{ Económico } & Desempeño económico & \\
\hline & Presencia en el mercado & Transparencia en los fondos públicos \\
\hline & Consecuencias económicas indirectas & \\
\hline & Prácticas de adquisición & Criterios y prácticas de adquisición \\
\hline \multirow{10}{*}{ Ambiental } & Materiales & \\
\hline & Energía & \\
\hline & Agua & \\
\hline & Biodiversidad & \\
\hline & Emisiones & \\
\hline & Efluentes y residuos & \\
\hline & Productos y servicios & \\
\hline & Cumplimiento regulatorio & \\
\hline & Transporte & \\
\hline & General & \\
\hline \multirow{15}{*}{ Social } & Empleo & \\
\hline & Relaciones entre los trabajadores y la dirección & \\
\hline & Salud y seguridad en el trabajo & \\
\hline & Capacitación y educación & \\
\hline & Diversidad e igualdad de oportunidades & \\
\hline & Igualdad de retribución entre mujeres y hombres & \\
\hline & No discriminación & \\
\hline & Libertad de asociación y negociación colectiva & \\
\hline & Trabajo infantil & \\
\hline & Trabajo forzoso & \\
\hline & Derechos de la población indígena & \\
\hline & Comunidades locales & \\
\hline & Lucha contra la corrupción & Corrupción \\
\hline & Política pública & Posiciones de política pública \\
\hline & & Norma de Calidad del servicio \\
\hline
\end{tabular}

Fuente: Elaboración propia a partir de Global Reporting Initiative (2005 y 2013)

Anexo 2. Funciones del sector público en la RS

\begin{tabular}{|c|c|c|c|}
\hline Función & Legislación & Control & Mecanismos \\
\hline Obligar & $\begin{array}{l}\text { Legislación para ordenar } \\
\text { y controlar }\end{array}$ & Regulación e inspección & $\begin{array}{l}\text { Sanciones e incentivos legales y } \\
\text { fiscales }\end{array}$ \\
\hline \multirow{2}{*}{ Facilitar } & Legislación facilitadora & Creación de incentivos & Capacitación "Creación de capacidad" \\
\hline & Apoyo financiero & Sensibilización & Estimulación de los mercados \\
\hline Colaborar & Combinación de recursos & $\begin{array}{l}\text { Compromiso con los } \\
\text { stakeholders }\end{array}$ & Diálogo \\
\hline Promocionar & Apoyo político & & Difusión y reconocimiento \\
\hline
\end{tabular}

Fuente: Fox et al. (2002) y García et al. (2013). 